\begin{tabular}{|r|l|}
\hline \multicolumn{2}{|c|}{ Statistica Sinica Preprint No: SS-2021-0253 } \\
\hline Title & $\begin{array}{l}\text { Score Tests With Incomplete Covariates and } \\
\text { High-Dimensional Auxiliary Variables }\end{array}$ \\
\hline Manuscript ID & SS-2021-0253 \\
\hline URL & http://www.stat.sinica.edu.tw/statistica/ \\
\hline DOI & $10.5705 /$ ss.202021.0253 \\
\hline Complete List of Authors & $\begin{array}{l}\text { Kin Yau Wong and } \\
\text { Jiahui Feng }\end{array}$ \\
\hline Corresponding Author & Kin Yau Wong \\
\hline E-mail & kin-yau.wong@polyu.edu.hk \\
\hline Notice: Accepted version subject to English editing.
\end{tabular}




\title{
SCORE TESTS WITH INCOMPLETE COVARIATES AND HIGH-DIMENSIONAL AUXILIARY VARIABLES
}

\author{
Kin Yau Wong and Jiahui Feng \\ The Hong Kong Polytechnic University
}

\begin{abstract}
Analysis of modern biomedical data is often complicated by the presence of missing values. When variables of interest are missing for some subjects, it is desirable to use observed auxiliary variables, which are sometimes high-dimensional, to impute or predict the missing values to improve statistical efficiency. Although many methods have been developed for prediction using high-dimensional variables, it is challenging to perform valid inference based on the predicted values. In this paper, we develop an association test for an outcome variable and a potentially missing covariate, where the covariate can be predicted using selected variables from a set of highdimensional auxiliary variables. We establish the validity of the test under data-driven model selection procedures. We demonstrate the validity of the proposed method and its advantages over existing methods through extensive simulation studies and provide an application to a major cancer genomics study.
\end{abstract}

Key words and phrases: Association test; Integrative analysis; Missing data; Post-selection inference; Variable selection.

\section{Introduction}

In many clinical and epidemiological studies, investigators are interested in testing the presence of association between an outcome variable and covariates of interest. In practice, such association analyses are often complicated by missing data, arising due to cost 
or other constraints. The problem of missing data is especially prevalent in large-scale genomic studies, where multiple types of genomic data are collected on a large number of subjects, often over different locations and time periods. For example, in The Cancer Genome Atlas (TCGA) (https://cancergenome.nih.gov/), over 10,000 subjects with 33 cancer types were measured for multiple types of genomic data, including DNA alterations, RNA expressions, and protein expressions, but protein expressions were not measured for a substantial number of subjects. As another example, in the Trans-Omics for Precision Medicine (TOPMed) program (https://www.nhlbi.nih.gov/research/resources/ nhlbi-precision-medicine-initiative/topmed), whole-genome sequencing data are available on hundreds of thousands of subjects, but other types of genomic data, such as RNA sequencing, methylation, and metabolites, are available for only tens of thousands of subjects or fewer.

A naive approach to handle missing data is to perform a complete-case analysis, where subjects with missing data are discarded. Such an approach is obviously inefficient when subjects with missing data were measured on some relevant auxiliary variables, because information of the auxiliary variables would be discarded. An alternative approach is imputation, where the missing values are imputed by plausible values based on the observed data, and conventional methods are then applied to the imputed data set. However, although estimation based on imputed data may be more efficient than a complete-case analysis, conventional inferential procedures based on (singly) imputed data are generally invalid. More sophisticated statistical methods to handle missing data involve modeling the missing-data mechanism or the variables with missing values; see Little and Rubin (2019) for detailed discussions. 
Although regression analysis with missing data has been extensively studied, association testing for incomplete data has received relatively less attention. To perform association tests with missing genotype data, $\mathrm{Hu}$ et al. (2015) considered the score test based on imputed genotype data and proposed a variance estimator that properly accounts for the differential quality between observed and imputed genotypes. Under outcome-dependent sampling designs, Derkach, Lawless and Sun (2015) and Lawless (2018) proposed to model the variable with missing values and studied the score test based on the full likelihood. Under extreme phenotype sampling designs in genetic association studies, Bjørnland et al. (2018) considered a similar model-based score test and a complete-case score test based on the conditional likelihood given the sampling mechanism. Wong, Zeng and Lin (2019) proposed to model the variable with missing values semiparametrically and developed a score test that is robust against misspecification of the missing-variable model. In all existing work, the observed variables are low-dimensional, and the methods cannot be readily extended to accommodate high-dimensional data.

In this paper, we consider the association test between an outcome of interest and an incomplete covariate, where the incomplete covariate may be associated with potentially high-dimensional auxiliary variables. We consider a missing-at-random scenario, where the missing mechanism may depend on the outcome of interest and observed covariates, and a complete-case analysis or a simple imputation approach is generally invalid. We propose to select a subset of auxiliary variables and fit a regression model of the covariate of interest against the selected variables. Then, we perform the score test for the covariate effect in the outcome model under the full likelihood, which includes both the outcome and covariate models. We show that the proposed procedure, though derived by assuming 
a prespecified covariate model, is valid even when the selection event of the auxiliary variables is random.

The current problem is inherently a post-selection inference problem, where we wish to perform inference using a model selected based on the observed data. It is wellknown that in general, conventional inferential procedures, such as the $F$ test and the $t$ test, on a selected model are invalid, because the parameters to be estimated or tested arise from a data-driven model selection procedure and are "random". There is a rapidgrowing literature on post-selection inference. One approach is to perform conditional inference for the model parameters given the model selection event (Lee et al., 2016; Tibshirani et al., 2016; Heller et al., 2018; Tian, Loftus and Taylor, 2018). This approach is dependent on distributional assumptions and is applicable only when the model is selected based on a prespecified formal selection procedure, such as forward selection or lasso. An alternative approach is to develop uniformly valid inferential procedures that can be used after arbitrary model selection (Berk et al., 2013; Bachoc, Leeb and Pötscher, 2019; Kuchibhotla et al. 2020). Such procedures are based on uniform tail probability inequalities and thus are often conservative.

The proposed approach is akin to the uniform approach, which is not restricted to a specific model selection procedure, and no assumptions on the correctness of the selected model are made. Nevertheless, an essential difference between the current framework and those considered in the literature is that the selected model, that is, the covariate model, is only of secondary interest, and the parameter of interest does not vary with the selected model. As a result of this special structure, the variability of model selection does not affect the asymptotic distribution of the score statistic. Therefore, unlike existing 
methods that are potentially conservative, the proposed score test is as efficient as the standard score test that treats the selected covariate model as prespecified.

There is a related literature on high-dimensional inference based on debiased estimators or decorrelated score functions van de Geer et al., 2014; Zhang and Zhang, 2014; Ning and Liu, 2017). In these approaches, the target of inference is the parameters in the full, high-dimensional regression model; this is in contrast with post-selection approaches, which focus on the inference of a selected model. One may prefer these high-dimensional approaches because the full model is considered more scientifically relevant than the selected model. Nevertheless, in the current framework, this potential advantage is not pertinent, because the high-dimensional model is only of secondary interest, and the parameter of interest remains the same regardless of whether the full or reduced model is fit. Because these high-dimensional approaches require sparsity assumptions on the true model and involve selection of multiple tuning parameters, they are not considered in this paper.

The rest of this paper is structured as follows. In Section 2, we formulate the model and develop a post-selection score test. In Section 3, we establish the asymptotic properties of the proposed score test. In Section 4, we report the results from simulation studies. In Section 5, we provide an application to a data set from TCGA. We make some concluding remarks in Section 6 and relegate technical details to the Appendix.

\section{Model and the post-selection score test}

Consider an outcome of interest $Y$, a covariate of interest $S$, a vector of other covariates $\boldsymbol{X}$, and a potentially high-dimensional vector of auxiliary variables $\boldsymbol{A}$. For example, in 
genomic studies, $Y$ may represent a disease phenotype, $S$ may represent a genomic variable of interest, $\boldsymbol{X}$ may represent clinical or demographic variables, and $\boldsymbol{A}$ may represent other types of genomic or environmental variables collected in the study. The vector of covariates $\boldsymbol{X}$ includes a constant component of 1. Assume that

$$
Y \mid(\boldsymbol{X}, S) \sim F_{Y}\left(; \boldsymbol{\alpha}^{\mathrm{T}} \boldsymbol{X}+\beta S\right),
$$

where $\boldsymbol{\alpha}$ and $\beta$ are regression parameters, and $F_{Y}$ is a distribution function such that for some known function $\mu(\cdot), \mathrm{E}\left[\left\{Y-\mu\left(\boldsymbol{\alpha}^{\mathrm{T}} \boldsymbol{X}+\beta S\right)\right\}\left(\boldsymbol{X}^{\mathrm{T}}, S\right)^{\mathrm{T}}\right]=\mathbf{0}$ at the true values of $\boldsymbol{\alpha}$ and $\beta$. This formulation includes as special cases the linear regression model, with $\mu(x)=x$, and the logistic regression model, with $\mu(x)=e^{x} /\left(1+e^{x}\right)$. The parameter $\beta$ captures the effect of $S$ on $Y$ given $\boldsymbol{X}$. In cancer genomic studies, we typically set $\boldsymbol{X}$ to be clinical or demographic variables and do not include mediator variables in the effect of $S$ on $Y$ (such as downstream variables of $S$ ) in $\boldsymbol{X}$. In this case, $\beta$ represents the total effect of $S$ after accounting for clinical/demographic covariates. We do not assume an explicit model for $S$ but allow an arbitrary association structure with $(\boldsymbol{X}, \boldsymbol{A})$. Because the major purpose of fitting the model of $S$ is to predict missing $S$ values, we can set $\boldsymbol{A}$ to be (potential) predictive variables of $S$.

Suppose that $S$ may be missing, and let $R$ be the indicator of whether $S$ is observed. Specifically, $R=1$ if $S$ is observed, and $R=0$ if otherwise. We assume that $R$ is conditionally independent of $(S, \boldsymbol{A})$ given $(Y, \boldsymbol{X})$. This missing mechanism is common in two-phase studies, where the outcome $Y$ and basic covariates $\boldsymbol{X}$ are measured for all subjects in the first phase, and subjects with certain outcome or covariate values are selected to be measured for an expensive covariate $S$ in the second phase. We do not allow 
$R$ to depend directly on $\boldsymbol{A}$, because the auxiliary variables, though completely observed, may not be selected into the model of $S$. If $R$ depends on a component of $\boldsymbol{A}$ that is associated with $S$ and is not selected, then the missing mechanism becomes not at random. For a sample of size $n$, the observed data consist of $\left\{\left(Y_{i}, \boldsymbol{X}_{i}, \boldsymbol{A}_{i}, R_{i} S_{i}, R_{i}\right): i=1, \ldots, n\right\}$. The assumed relationships among these variables are illustrated in Figure 1.

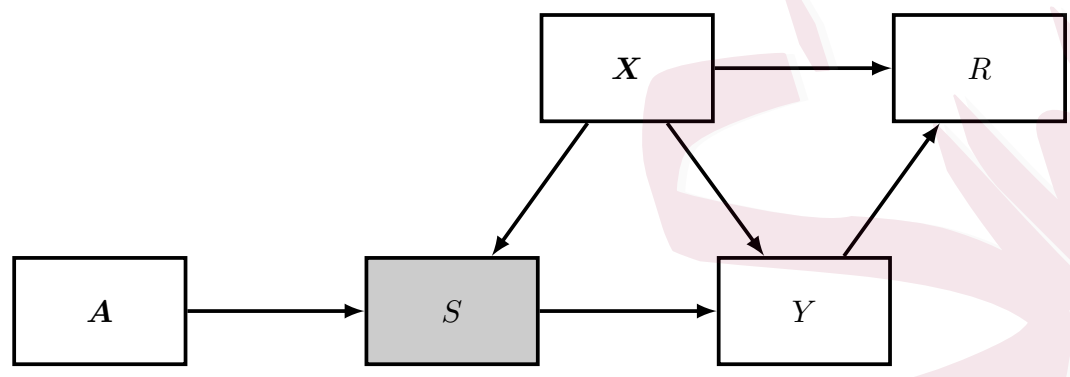

Figure 1: Relationships among the observed and incomplete variables.

We wish to test the null hypothesis $H_{0}: \beta=0$. Due to the presence of missing data, we propose to fit a working model of $S$ on $(\boldsymbol{X}, \boldsymbol{A})$ and adopt the score test based on the full model (including both the models of $Y$ and $S$ ). Fitting a working model of $S$ against $(\boldsymbol{X}, \boldsymbol{A})$ allows us to utilize information about the missing $S$ values contained in the auxiliary variables and is generally more efficient than ignoring the auxiliary variables. The score test is considered, instead of the Wald test and the likelihood-ratio test, because it only involves estimation under the null hypothesis, whereas the other two tests involve estimation under the alternative hypothesis. Note that estimation under the alternative hypothesis is more challenging because the likelihood generally involves an integration without a closed-form expression.

Because $\boldsymbol{A}$ is potentially high-dimensional, maximum likelihood estimation for the model of $S$ may be infeasible. Also, the model of $S$ is only of secondary interest, so 
full specification of the model may not be necessary. Therefore, we propose a two-step approach, where in the first step, we select a low-dimensional subset of $\boldsymbol{A}$ into the model of $S$, and in the second step, we perform a score test based on the model of $Y$ and a working model of $S$.

In the first step, we perform variable selection on $\boldsymbol{A}$. Let $\mathcal{K}^{*}$ be a general model selection operator, such that for an $m$-vector of outcome variables $\mathcal{Y}$ and an $(m \times p)$ matrix of covariates $\mathcal{Z}, \mathcal{K}^{*}(\mathcal{Y}, \mathcal{Z}): \mathbb{R}^{m} \times \mathbb{R}^{m \times p} \rightarrow \mathcal{C}_{p}$, where $\mathcal{C}_{p}$ is the collection of subsets of $\{1, \ldots, p\}$. For example, for marginal screening (Fan and Lv, 2008; Fan and Song, 2010) with a quantitative outcome variable and standardized $\mathcal{Z}, \mathcal{K}^{*}$ can be defined as $\mathcal{K}^{*}:(\mathcal{Y}, \mathcal{Z}) \mapsto\left\{j:\left|\mathcal{Y}^{\mathrm{T}} \mathcal{Z}_{j}\right|>\lambda\right\}$, where $\lambda$ is a tuning parameter, and $\mathcal{Z}_{j}$ is the $j$ th column of $\mathcal{Z}$. Likewise, for lasso (Tibshirani, 1996),

$$
\mathcal{K}^{*}:(\mathcal{Y}, \mathcal{Z}) \mapsto\left\{j: \widehat{\gamma}_{j} \neq 0, \text { where }\left(\widehat{\gamma}_{1}, \ldots, \widehat{\gamma}_{p}\right)^{\mathrm{T}}=\underset{\gamma}{\arg \min }\left(\|\mathcal{Y}-\mathcal{Z} \gamma\|^{2}+\lambda\|\gamma\|_{1}\right)\right\} .
$$

We use this operator to select a model for $S$ based on the residual $S-\widehat{\boldsymbol{\gamma}}_{X}^{\mathrm{T}} \boldsymbol{X}$ and $\boldsymbol{A}$, where $\widehat{\gamma}_{X} \equiv\left(\sum_{i=1}^{n} R_{i} \boldsymbol{X}_{i} \boldsymbol{X}_{i}^{\mathrm{T}}\right)^{-1} \sum_{i=1}^{n} R_{i} \boldsymbol{X}_{i} S_{i}$ is the least-squares estimator of $S$ on $\boldsymbol{X}$ using the subjects with $R=1$. The selected components of $\boldsymbol{A}$ are $\mathcal{K}^{*}\left(\mathcal{S}-\boldsymbol{X} \widehat{\boldsymbol{\gamma}}_{X}, \boldsymbol{A}\right)$, where $\mathcal{S}$ is a vector that consists of $\left\{S_{i}: R_{i}=1\right\}$, and $\boldsymbol{X}$ and $\boldsymbol{A}$ are matrices that consist of rows of $\left\{\boldsymbol{X}_{i}: R_{i}=1\right\}$ and $\left\{\boldsymbol{A}_{i}: R_{i}=1\right\}$, respectively. For simplicity of presentation, we write $\mathcal{K}^{*}=\mathcal{K}^{*}\left(\mathcal{S}-\boldsymbol{X} \widehat{\gamma}_{X}, \boldsymbol{A}\right)$ and let $\mathcal{K}$ be the observed value of $\mathcal{K}^{*}$.

Let $\boldsymbol{W}_{\mathcal{K}}$ denote the vector that consists of $\boldsymbol{X}$ and the components of $\boldsymbol{A}$ indexed by $\mathcal{K}$. In the second step, we fit model 2.1 and the working model $S=\boldsymbol{\gamma}_{\mathcal{K}}^{\mathrm{T}} \boldsymbol{W}_{\mathcal{K}}+\delta$ under the null hypothesis $H_{0}$, where $\delta$ is a mean-zero error term, and $\gamma_{\mathcal{K}}$ is a vector of regression parameters. In particular, we estimate $\boldsymbol{\gamma}_{\mathcal{K}}$ by $\widehat{\boldsymbol{\gamma}}_{\mathcal{K}} \equiv\left(\sum_{i=1}^{n} R_{i} \boldsymbol{W}_{\mathcal{K}, i} \boldsymbol{W}_{\mathcal{K}, i}^{\mathrm{T}}\right)^{-1} \sum_{i=1}^{n} R_{i} \boldsymbol{W}_{\mathcal{K}, i} S_{i}$, 
the least-squares estimator using the subjects with observed $S$ values. Let $\widehat{\boldsymbol{\alpha}}$ be the Zestimator of $\boldsymbol{\alpha}$ under $H_{0}$, such that $\sum_{i=1}^{n}\left\{Y_{i}-\mu\left(\widehat{\boldsymbol{\alpha}}^{\mathrm{T}} \boldsymbol{X}_{i}\right)\right\} \boldsymbol{X}_{i}=0$. The (scaled) score statistic for $\beta$ is

$$
U_{\beta}\left(\widehat{\boldsymbol{\alpha}}, \widehat{\boldsymbol{\gamma}}_{\mathcal{K}}\right)=\frac{1}{n^{1 / 2}} \sum_{i=1}^{n}\left\{Y_{i}-\mu\left(\widehat{\boldsymbol{\alpha}}^{\mathrm{T}} \boldsymbol{X}_{i}\right)\right\}\left\{R_{i} S_{i}+\left(1-R_{i}\right) \widehat{\boldsymbol{\gamma}}_{\mathcal{K}}^{\mathrm{T}} \boldsymbol{W}_{\mathcal{K}, i}\right\}
$$

Note that this coincides with the imputation-based score statistic, that is, the score statistic when the missing values of $S$ are imputed by the estimated mean $\widehat{\gamma}_{\mathcal{K}}^{\mathrm{T}} \boldsymbol{W}_{\mathcal{K}}$.

To obtain an asymptotic size $\alpha$ test, we need to derive the asymptotic distribution of $U_{\beta}\left(\widehat{\boldsymbol{\alpha}}, \widehat{\gamma}_{\mathcal{K}^{*}}\right)$ under $H_{0}$. This is highly challenging, because the model selection event $\left\{\mathcal{K}^{*}=\mathcal{K}\right\}$ is random, and the usual arguments based on the Taylor's series expansion of the score statistic do not apply. Nevertheless, as we establish in Section $3, U_{\beta}\left(\widehat{\boldsymbol{\alpha}}, \widehat{\gamma}_{\mathcal{K}^{*}}\right)$, properly scaled by a variance term that can be consistently estimated by an empirical sum-of-squares estimator, is asymptotically normal. In particular, the variance term resembles that derived based on the usual Taylor's series expansion on the score statistic. Let $\boldsymbol{\alpha}_{0}$ be the true value of $\boldsymbol{\alpha}$, and for a given selected model $\mathcal{K}$, define $\boldsymbol{\gamma}_{0 \mathcal{K}} \equiv \arg \min _{\boldsymbol{\gamma}} \mathrm{E}\left\{R\left(S-\boldsymbol{\gamma}^{\mathrm{T}} \boldsymbol{W}_{\mathcal{K}}\right)^{2}\right\}$ as the true value of $\boldsymbol{\gamma}_{\mathcal{K}}$. Let $\boldsymbol{I}_{\alpha \alpha}=$ $\mathrm{E}\left\{\mu^{\prime}\left(\boldsymbol{\alpha}_{0}^{\mathrm{T}} \boldsymbol{X}\right) \boldsymbol{X} \boldsymbol{X}^{\mathrm{T}}\right\}, \boldsymbol{I}_{\beta \alpha}=-\mathrm{E}\left[\mu^{\prime}\left(\boldsymbol{\alpha}_{0}^{\mathrm{T}} \boldsymbol{X}\right) \boldsymbol{X}\left\{R S+(1-R) \boldsymbol{\gamma}_{0 \mathcal{K}}^{\mathrm{T}} \boldsymbol{W}_{\mathcal{K}}\right\}\right], \boldsymbol{I}_{\gamma \gamma}=\mathrm{E}\left(R \boldsymbol{W}_{\mathcal{K}} \boldsymbol{W}_{\mathcal{K}}^{\mathrm{T}}\right)$, and $\boldsymbol{I}_{\beta \gamma}=\mathrm{E}\left[\left\{Y-\mu\left(\boldsymbol{\alpha}_{0}^{\mathrm{T}} \boldsymbol{X}\right)\right\}(1-R) \boldsymbol{W}_{\mathcal{K}}\right]$, where $\mu^{\prime}$ denotes the first derivative of $\mu$. If the model $\mathcal{K}$ is prespecified, then the Taylor's series expansion of $U_{\beta}\left(\widehat{\boldsymbol{\alpha}}, \widehat{\gamma}_{\mathcal{K}}\right)$ at $\left(\boldsymbol{\alpha}_{0}, \boldsymbol{\gamma}_{0 \mathcal{K}}\right)$ yields

$$
\begin{gathered}
U_{\beta}\left(\widehat{\boldsymbol{\alpha}}, \widehat{\boldsymbol{\gamma}}_{\mathcal{K}}\right)=\frac{1}{n^{1 / 2}} \sum_{i=1}^{n}\left[\left\{Y_{i}-\mu\left(\alpha_{0}^{\mathrm{T}} \boldsymbol{X}_{i}\right)\right\}\left\{R_{i} S_{i}+\left(1-R_{i}\right) \boldsymbol{\gamma}_{0 \mathcal{K}}^{\mathrm{T}} \boldsymbol{W}_{\mathcal{K}, i}+\boldsymbol{I}_{\beta \alpha}^{\mathrm{T}} \boldsymbol{I}_{\alpha \alpha}^{-1} \boldsymbol{X}_{i}\right\}\right. \\
\left.+\boldsymbol{I}_{\beta \gamma}^{\mathrm{T}} \boldsymbol{I}_{\gamma \gamma}^{-1} \boldsymbol{W}_{\mathcal{K}, i} R_{i}\left(S_{i}-\gamma_{0 \mathcal{K}}^{\mathrm{T}} \boldsymbol{W}_{\mathcal{K}, i}\right)\right]+o_{p}(1)
\end{gathered}
$$


under regularity conditions. Based on this expansion, we can estimate the asymptotic variance of $U_{\beta}\left(\widehat{\boldsymbol{\alpha}}, \widehat{\gamma}_{\mathcal{K}}\right)$ by $\widehat{\sigma}^{2}(\mathcal{K})=n^{-1} \sum_{i=1}^{n}\left\{\widehat{\sigma}_{i}(\mathcal{K})-\bar{\sigma}(\mathcal{K})\right\}^{2}$, where

$$
\begin{aligned}
\widehat{\sigma}_{i}(\mathcal{K})= & \left\{Y_{i}-\mu\left(\widehat{\boldsymbol{\alpha}}^{\mathrm{T}} \boldsymbol{X}_{i}\right)\right\}\left\{R_{i} S_{i}+\left(1-R_{i}\right) \widehat{\boldsymbol{\gamma}}_{\mathcal{K}}^{\mathrm{T}} \boldsymbol{W}_{\mathcal{K}, i}+\widehat{\boldsymbol{I}}_{\beta \alpha}^{\mathrm{T}} \widehat{\boldsymbol{I}}_{\alpha \alpha}^{-1} \boldsymbol{X}_{i}\right\} \\
& +\widehat{\boldsymbol{I}}_{\beta \gamma}^{\mathrm{T}} \widehat{\boldsymbol{I}}_{\gamma \gamma}^{-1} \boldsymbol{W}_{\mathcal{K}, i} R_{i}\left(S_{i}-\widehat{\boldsymbol{\gamma}}_{\mathcal{K}}^{\mathrm{T}} \boldsymbol{W}_{\mathcal{K}, i}\right),
\end{aligned}
$$

$\bar{\sigma}(\mathcal{K})=n^{-1} \sum_{i=1}^{n} \widehat{\sigma}_{i}(\mathcal{K})$, and $\widehat{\boldsymbol{I}}_{\alpha \alpha}, \widehat{\boldsymbol{I}}_{\beta \alpha}, \widehat{\boldsymbol{I}}_{\beta \gamma}$, and $\widehat{\boldsymbol{I}}_{\gamma \gamma}$, are the empirical counterparts of $\boldsymbol{I}_{\alpha \alpha}, \boldsymbol{I}_{\beta \alpha}, \boldsymbol{I}_{\beta \gamma}$, and $\boldsymbol{I}_{\gamma \gamma}$, respectively, with the expectations replaced by empirical means and true parameters replaced by estimators. We can show that even though this variance term is derived based on fixed $\mathcal{K}, U_{\beta}\left(\widehat{\boldsymbol{\alpha}}, \widehat{\gamma}_{\mathcal{K}^{*}}\right) / \widehat{\sigma}\left(\mathcal{K}^{*}\right)$ converges to the standard normal distribution under $H_{0}$. Therefore, for an asymptotic size $\alpha$ test, we reject $H_{0}$ if $U_{\beta}\left(\widehat{\boldsymbol{\alpha}}, \widehat{\gamma}_{\mathcal{K}^{*}}\right)^{2} / \widehat{\sigma}^{2}\left(\mathcal{K}^{*}\right)>\chi_{1, \alpha}^{2}$

The proposed test does not require correct specification of the models of $Y$ and $S$. For the outcome model, we only require that $\mathrm{E}\left[\left\{Y-\mu\left(\boldsymbol{\alpha}_{0}^{\mathrm{T}} \boldsymbol{X}\right)\right\}\left(\boldsymbol{X}^{\mathrm{T}}, S\right)^{\mathrm{T}}\right]=\mathbf{0}$ under the null hypothesis, because an empirical variance estimator is used instead of a model-based estimator. For the covariate model, as detailed in Section 3, we require the association structure between $S$ and $\boldsymbol{X}$ to be correctly specified but allow arbitrary association between $S$ and $\boldsymbol{A}$; correct specification of the association between $S$ and $\boldsymbol{X}$ is generally needed (Derkach, Lawless and Sun, 2015, Lawless, 2018). The association structure between $S$ and $\boldsymbol{A}$ affects the power of the test but not its validity under the null hypothesis. 


\section{Asymptotic properties of the post-selection score test}

For any $\mathcal{K}$, let $\gamma_{0 X}$ and $\gamma_{0 A, \mathcal{K}}$ be the subvectors of $\gamma_{0 \mathcal{K}}$ that correspond to $\boldsymbol{X}$ and the selected components of $\boldsymbol{A}$, respectively. Define

$$
\begin{aligned}
\sigma_{1}^{2}(\mathcal{K})=\operatorname{Var}[ & \left.\epsilon\left\{S+(1-R) \boldsymbol{\gamma}_{0 \mathcal{K}}^{\mathrm{T}} \boldsymbol{W}_{\mathcal{K}}+\boldsymbol{I}_{\beta \alpha}^{\mathrm{T}} \boldsymbol{I}_{\alpha \alpha}^{-1} \boldsymbol{X}\right\}\right] \\
\sigma_{2}^{2}(\mathcal{K})=\operatorname{Var}[ & \left(\boldsymbol{\gamma}_{0 X}^{\mathrm{T}}+\boldsymbol{I}_{\beta \alpha}^{\mathrm{T}} \boldsymbol{I}_{\alpha \alpha}^{-1}\right)\{\mathrm{E}(\epsilon \mid R, \boldsymbol{X}) \boldsymbol{X}-\mathrm{E}(\epsilon \boldsymbol{X} \mid R)\} \\
+ & \{\mathrm{E}(\epsilon \mid R, \boldsymbol{X})-\mathrm{E}(\epsilon \mid R)\} \boldsymbol{\gamma}_{0 A, \mathcal{K}}^{\mathrm{T}} \boldsymbol{A}_{\mathcal{K}} \\
+ & \left.\left\{\mathrm{E}(\epsilon \mid R, \boldsymbol{X})+\boldsymbol{I}_{\beta \gamma}^{\mathrm{T}} \boldsymbol{I}_{\gamma \gamma}^{-1} \boldsymbol{W}_{\mathcal{K}}\right\} R\left(S-\boldsymbol{\gamma}_{0 \mathcal{K}}^{\mathrm{T}} \boldsymbol{W}_{\mathcal{K}}\right)\right] \\
\sigma_{3}^{2}(\mathcal{K})=\operatorname{Var}\{ & \left.\left(\boldsymbol{\gamma}_{0 X}^{\mathrm{T}}+\boldsymbol{I}_{\beta \alpha}^{\mathrm{T}} \boldsymbol{I}_{\alpha \alpha}^{-1}\right) \mathrm{E}(\epsilon \boldsymbol{X} \mid R)+\mathrm{E}(\epsilon \mid R) \boldsymbol{\gamma}_{0 A, \mathcal{K}}^{\mathrm{T}} \boldsymbol{A}_{\mathcal{K}}\right\},
\end{aligned}
$$

where $\epsilon=Y-\mu\left(\boldsymbol{\alpha}_{0}^{\mathrm{T}} \boldsymbol{X}\right)$, and let $\sigma^{2}(\mathcal{K})=\sum_{k=1}^{3} \sigma_{k}^{2}(\mathcal{K})$. Let $\|\cdot\|_{\psi_{\xi}}$ be an Orlicz norm, such that $\|X\|_{\psi_{\xi}}=\inf \left\{\eta>0: \mathrm{E}\left(e^{|X|^{\xi} / \eta^{\xi}}\right) \leq 2\right\}$, and $\|\cdot\|$ be the Euclidean norm. We assume the following conditions. Some conditions involve a generic positive constant $M$.

(C1) For some $\xi \in(0,2],\|Y\|_{\psi_{\xi}}+\|S\|_{\psi_{\xi}}+\max _{j}\left\|A_{j}\right\|_{\psi_{\xi}}<M$. The covariate $\boldsymbol{X}$ is bounded, so that $P(\|\boldsymbol{X}\|<M)=1$. Also, the estimator $\widehat{\boldsymbol{\alpha}}$ is strongly consistent un$\operatorname{der} \beta=0, \mu(\cdot)$ is twice continuously differentiable, and $\lambda_{\min }\left[\mathrm{E}\left\{\mu^{\prime}\left(\boldsymbol{\alpha}_{0}^{\mathrm{T}} \boldsymbol{X}\right) \boldsymbol{X} \boldsymbol{X}^{\mathrm{T}}\right\}\right]>$ $M^{-1}$, where $\lambda_{\min }(C)$ denotes the minimum eigenvalue of the matrix $C$.

(C2) There exists a sequence of collections of models $\Omega_{n}$, such that $P\left(\mathcal{K}^{*} \in \Omega_{n}\right) \rightarrow 1$, $\sup _{\mathcal{K} \in \Omega_{n}}|\mathcal{K}|=O\left(n^{\tau}\right)$, and $\log \left|\Omega_{n}\right|=O\left(n^{\kappa}\right)$, where $\tau$ and $\kappa$ are constants that satisfy $\tau<4 \xi /(5 \xi+12), 5 \tau / 4+3 \kappa / \xi<1$, and $\tau+4 \kappa / \xi<1$, and $|\mathcal{C}|$ denotes the cardinality of the set $\mathcal{C}$. Also, $\inf _{\mathcal{K} \in \Omega_{n}} \lambda_{\min }\left\{\mathrm{E}\left(R \boldsymbol{W}_{\mathcal{K}} \boldsymbol{W}_{\mathcal{K}}^{\mathrm{T}}\right)\right\}>M^{-1}, \sup _{\mathcal{K} \in \Omega_{n}} \mathrm{E}\left\{\left(\boldsymbol{\gamma}_{0 \mathcal{K}}^{\mathrm{T}} \boldsymbol{W}_{\mathcal{K}}\right)^{4}\right\}<$ $M$, and $\inf _{\mathcal{K} \in \Omega_{n}} \sigma^{2}(\mathcal{K})>M^{-1}$

(C3) The probability $P(R=1 \mid Y, \boldsymbol{X})>M^{-1}$ almost surely. 
(C4) Under $\beta=0$, the residual $\left(S-\gamma_{0 X}^{\mathrm{T}} \boldsymbol{X}\right)$ and $\boldsymbol{X}$ are independent, and $\boldsymbol{A}$ is independent of $(Y, \boldsymbol{X})$.

(C5) The models selected based on the estimated residuals $\left(S_{i}-\widehat{\gamma}_{X}^{\mathrm{T}} \boldsymbol{X}_{i}\right)_{i: R_{i}=1}$ and the actual residuals $\left(S_{i}-\gamma_{0 X}^{\mathrm{T}} \boldsymbol{X}_{i}\right)_{i: R_{i}=1}$ are such that

$$
P\left\{\mathcal{K}^{*}\left(\mathcal{S}-\mathcal{X} \widehat{\gamma}_{X}, \mathcal{A}\right) \neq \mathcal{K}^{*}\left(\mathcal{S}-\mathcal{X} \gamma_{0 X}, \mathcal{A}\right)\right\}=o(1)
$$

and

$$
\sup _{\mathcal{K} \in \Omega_{n}} \frac{P\left\{\mathcal{K}^{*}\left(\mathcal{S}-\mathcal{X} \widehat{\gamma}_{X}, \mathcal{A}\right)=\mathcal{K}\right\}}{P\left\{\mathcal{K}^{*}\left(\mathcal{S}-\mathcal{X} \gamma_{0 X}, \mathcal{A}\right)=\mathcal{K}\right\}}<M
$$

(C6) For a random sample of size $m$, let $\widetilde{\mathcal{S}}=\left(S_{1}, \ldots, S_{m}\right)^{\mathrm{T}}, \widetilde{\mathcal{X}}=\left(\boldsymbol{X}_{1}, \ldots, \boldsymbol{X}_{m}\right)^{\mathrm{T}}$, and $\widetilde{\mathcal{A}}=\left(\boldsymbol{A}_{1}, \ldots, \boldsymbol{A}_{m}\right)^{\mathrm{T}}$. The random variable

$$
\sup _{\mathcal{K} \in \Omega_{m}}\left|\frac{P\left\{\mathcal{K}^{*}\left(\widetilde{\mathcal{S}}-\widetilde{\mathcal{X}} \gamma_{0 X}, \widetilde{\mathcal{A}}\right)=\mathcal{K} \mid \widetilde{\mathcal{A}}\right\}}{P\left\{\mathcal{K}^{*}\left(\widetilde{\mathcal{S}}-\widetilde{\mathcal{X}} \gamma_{0 X}, \widetilde{\mathcal{A}}\right)=\mathcal{K}\right\}}-1\right|
$$

converges to 0 in mean as $m \rightarrow \infty$.

Remark 1. Condition (C1) imposes constraints on the tail probabilities of the observed variables. With $\xi=1$ or $\xi=2$, we assume each component of $(Y, S, \boldsymbol{A})$ to be subexponential or sub-Gaussian, respectively. To maintain a flexible model for $Y$, we assume that $\boldsymbol{X}$ is bounded. Desired theoretical results could be obtained by only requiring $\max _{j}\left\|X_{j}\right\|_{\psi_{\xi}}<M$, but additional conditions on $\mu$ would be required. Condition (C2) allows the set of "possibly-selected models" $\Omega_{n}$ to grow exponentially with $n$ and the size of the selected model to increase at a polynomial rate of $n$. For example, for $\xi=$ 2, we allow $\sup _{\mathcal{K} \in \Omega_{n}}|\mathcal{K}|=O\left(n^{1 / 4}\right)$ and $\left|\Omega_{n}\right|=O\left\{\exp \left(n^{1 / 4}\right)\right\}$. Note that if the model selection procedure yields consistent selection, then $\Omega_{n}$ can be chosen to be a singleton 
set, consisting only of the true model. In our setting, we allow the model selection event to be genuinely random even when $n$ increases to infinity. Condition (C3) ensures that a nonvanishing portion of subjects have observed $S$.

Remark 2. Condition (C4) requires that $S$ exhibits a linear association structure with $\boldsymbol{X}$ and that $(Y, \boldsymbol{X})$ are independent of the auxiliary variables. This guarantees that $(Y, \boldsymbol{X})$ are independent of the model selection event, which is based on the residuals in the model of $S$ and the auxiliary variables. In cancer genomic studies where $\boldsymbol{X}$ represents demographic variables and $\boldsymbol{A}$ represents genomic variables (such as gene expressions in the tumor), $\boldsymbol{X}$ and $\boldsymbol{A}$ are plausibly independent. In general, because $\boldsymbol{X}$ is low-dimensional, the independence between $\boldsymbol{A}$ and $\boldsymbol{X}$ can be (approximately) achieved by projecting components of $\boldsymbol{A}$ to the orthogonal complement of the span of $\boldsymbol{X}$ or functions of $\boldsymbol{X}$. The independence between $\boldsymbol{A}$ and $Y$ can be relaxed to allow some auxiliary variables that are not associated with $S$ to depend on $Y$; the technical formulation of the relaxed condition is deferred to Appendix 1. For marginal screening, the relaxed condition allows the auxiliary variables not in any models in $\Omega_{n}$ to depend on $Y$ (and $\boldsymbol{X}$ ). Requiring the (potentially) selected auxiliary variables to be independent of $Y$ is quite reasonable under the null hypothesis, because these variables are generally associated with $S$. If they are also associated with $Y$, then except at some specific parameter values, $S$ and $Y$ would be marginally associated, and the null hypothesis does not hold.

Remark 3. Conditions (C5) and (C6) impose mild conditions on the model selection operator. Condition (C5) requires that the model selected based on the estimated residuals and that selected based on the actual residuals are asymptotically equal. This is easily 


\section{ASYMPTOTIC PROPERTIES OF THE POST-SELECTION SCORE TEST}

satisfied, because the least-squares estimator $\widehat{\gamma}_{X}$ is consistent. Condition (C6) requires that the marginal probability of selecting a model is asymptotically equal to the conditional probability of the same event given the auxiliary variables. This is true of common model selection operators, which select a model based on the association between the outcome and the covariates, and the covariates alone do not contain information about the model selection event. We discuss the verification of these conditions under a marginal screening procedure in the Supplementary Material.

We impose conditions on the number of possibly-selected models instead of the total number of auxiliary variables, because the former is directly relevant to the asymptotic distribution of the score statistic. Nevertheless, for a given maximal selected model size $q_{n} \equiv \sup _{\mathcal{K} \in \Omega_{n}}|\mathcal{K}|$, we have

$$
r_{n} \equiv\left|\Omega_{n}\right| \leq \sum_{s=1}^{q_{n}}\left(\begin{array}{c}
p_{n} \\
s
\end{array}\right) \leq\left(\frac{e p_{n}}{q_{n}}\right)^{q_{n}}
$$

where $p_{n}$ is the total number of auxiliary variables. The condition on $r_{n}$ is satisfied if $\log p_{n}=O\left(n^{\kappa-\tau}\right)$, with $\kappa$ and $\tau$ satisfying the inequalities in condition (C2). In fact, if most auxiliary variables are only weakly associated with $S$, then $r_{n}$ could be much smaller than the above upper bound.

We have the following results.

Theorem 1. Under conditions $(C 1)-(C 6)$ and $H_{0}, U_{\beta}\left(\widehat{\boldsymbol{\alpha}}, \widehat{\gamma}_{\mathcal{K}^{*}}\right) / \sigma\left(\mathcal{K}^{*}\right)$ converges weakly to the standard normal distribution.

Theorem 2. Under conditions (C1)-(C6) and $H_{0}$,

$$
\mathrm{E}\left\{\sup _{\mathcal{K} \in \Omega_{n}}\left|\widehat{\sigma}^{2}(\mathcal{K})-\sigma^{2}(\mathcal{K})\right|\right\}=o(1)
$$


Remark 4. Theorem 1 states that the scaled score statistic, which is derived from a randomly selected model, converges in distribution to a standard normal distribution marginally. A key step in the proof is to show that the score statistic can be (asymptotically) written as a sum of independent variables that are mean zero conditional on the model selection event and possibly other components of the observed data. Then, we can employ the Lindeberg approach to the proof of the central limit theorem to establish the desired result. Theorem 2 states that the scaling term of the score statistic in Theorem 1 can be uniformly consistently estimated by the proposed sum-of-squares estimator over the set of possibly-selected models $\Omega_{n}$.

An outline of the proof of Theorem 1 is given in Appendix 2, and the complete proofs of Theorems 1 and 2 are given in the Supplementary Material. Combining the above results, we have the following corollary.

Corollary 1. Under conditions $(C 1)-(C 6)$ and $H_{0}, U_{\beta}\left(\widehat{\boldsymbol{\alpha}}, \widehat{\gamma}_{\mathcal{K}^{*}}\right) / \widehat{\sigma}\left(\mathcal{K}^{*}\right)$ converges weakly to the standard normal distribution.

\section{Simulation studies}

Let $\boldsymbol{X}=\left(X_{1}, \ldots, X_{5}\right)^{\mathrm{T}}$, where $\left(X_{1}, X_{2}, X_{3}\right)$ are mean-zero multivariate normal variables with $\operatorname{Cov}\left(X_{j}, X_{k}\right)=0.5^{|j-k|}(j, k=1,2,3), X_{4} \sim \operatorname{Bernoulli}(0.1), X_{5} \sim \operatorname{Bernoulli}(0.2)$, and $X_{4}$ and $X_{5}$ are independent of each other and $\left(X_{1}, X_{2}, X_{3}\right)$. Let $\boldsymbol{A}$ be a $p$-vector of independent standard normal variables. We set $S=\boldsymbol{\gamma}_{X}^{\mathrm{T}} \boldsymbol{X}+\boldsymbol{\gamma}_{A}^{\mathrm{T}} \boldsymbol{A}+\boldsymbol{\gamma}_{A, 2}^{\mathrm{T}} \boldsymbol{A}^{2}+\delta$, where $\boldsymbol{A}^{2}$ is a $p$-vector of the squared components of $\boldsymbol{A}, \delta$ is standard normal, $\boldsymbol{\gamma}_{X}=(0.1, \ldots, 0.1)^{\mathrm{T}}$, and $\gamma_{A, 2}$ is 0.1 at the first 5 components and 0 elsewhere. We considered two different 
values of $\gamma_{A}$. In Setting 1, we set $\gamma_{A}$ to be 0.25 at the first 20 components and 0 at the remaining components, whereas in Setting 2, we set $\gamma_{A}$ to be 0.25 at the first 20 components, 0.02 at the subsequent 80 components, and 0 at the remaining components. In Setting 1, the model is sparse, and a small number of auxiliary variables have strong effects on $S$. In Setting 2, the model contains a mixture of strong and weak signals from the auxiliary variables.

We considered a quantitative and a binary outcome variable $Y$. For the quantitative outcome, we set $Y=\boldsymbol{\alpha}^{\mathrm{T}} \boldsymbol{X}+\beta S+\epsilon$, where $\epsilon$ is standard normal, and $\boldsymbol{\alpha}=$ $(1,-1,1,-1,1)^{\mathrm{T}}$. For the binary outcome, we set $\operatorname{logit}\{P(Y=1 \mid \boldsymbol{X}, S)\}=-2.2+$ $\boldsymbol{\alpha}^{\mathrm{T}} \boldsymbol{X}+\beta S$, where $\boldsymbol{\alpha}$ is the same as that under the linear model; the proportion of subjects with $Y=1$ is about $15-20 \%$. We considered two missing-data mechanisms. The first mechanism is missing completely at random (MCAR), where the missing-data status is independent of other variables. The second mechanism is missing at random (MAR), where for the quantitative outcome, an equal number of subjects at the two extreme tails of the distribution of $Y$ were selected to have observations on $S$, whereas for the binary outcome, all subjects with $Y=1$ were selected, and a fraction of subjects with $Y=0$ were selected to attain the desired missing proportion. We considered sample sizes of $n=500$ and 1000 and numbers of auxiliary variables of $p=200,500,1000,1500$, and 2000. For the alternative hypothesis, we set $\beta=2 n^{-1 / 2}$ and $6 n^{-1 / 2}$ for the quantitative and binary outcome variables, respectively. For each setting, we simulated 100,000 and 10,000 replicates for $\beta=0$ and $\beta \neq 0$, respectively.

We compared the performance of five tests: (1) the standard score test using complete data only; (2) the standard score test with missing data imputed under a working 
linear model of $S$ on $\boldsymbol{X}$ and components of $\boldsymbol{A}$ selected using marginal screening, where a component of $\boldsymbol{A}$ is selected if its absolute empirical correlation with $S-\widehat{\gamma}_{X}^{\mathrm{T}} \boldsymbol{X}$ among the subjects with complete data is larger than a certain threshold; (3) the score test based on the full likelihood with a working linear model of $S$ against $\boldsymbol{X}$ alone; (4) the proposed test, where the working model of $S$ is selected in the same way as (2); and (5) the score test based on the full likelihood with a linear model of $S$ against $\boldsymbol{X}$ and the components of $\boldsymbol{A}$ that are associated with $S$. We refer to methods (1)-(5) as the complete-case analysis, the simple imputation method, the covariate-only method, the proposed method, and the true model method, respectively. In the simple imputation, proposed, and true model methods, only first-order terms of $\boldsymbol{A}$ are in the working models, so none of the models is "correct". Nevertheless, according to our theory, the proposed method is still valid under such misspecification. For the simple imputation and proposed methods, the threshold for screening is selected using BIC. For the covariate-only and true model methods, the variance of the score statistic is estimated using the proposed empirical sum-of-squares estimator instead of the usual estimator based on the second derivative of the log-likelihood. This is for ease of comparison with the proposed method, and the two variance estimators are asymptotically equivalent. The true model method is a gold standard but is not practical, because it requires knowledge of the relevant predictors of $S$

The results under missing proportion of $60 \%$ are plotted in Figures 2 and 3 , and the results under missing proportion of $30 \%$ are plotted in Figures S1 and S2 of the Supplementary Material; the power of methods that inflate the type I error is not presented. The significance level is set to be 0.05 . Under missing at random and the linear outcome 
model, both the complete-case analysis and simple imputation method inflate the type I error, because they underestimate the variance of the score statistic. The covariateonly method and the true model method preserve the type I error; they do not involve model selection, and their validity follows from a conventional argument. The proposed method, despite involving model selection variability, preserves the type I error; in fact, under Setting 2, any given model is selected at most $0.006 \%$ and $3.804 \%$ of the times over all simulation replicates with sample size 500 and 1000, respectively. The pattern of results under missing at random and the binary outcome model are similar, but the complete-case analysis preserves the type I error due to the validity of inference based on the prospective likelihood under a case-control study and the logistic regression model (Prentice and Pyke, 1979). Under missing completely at random, all methods preserve the type I error.

Under the alternative hypothesis, the simple imputation method under missing at random has relatively high power due to underestimation of the variance of the score statistic; this is similar for the complete-case analysis under missing at random and the logistic outcome model. Under settings where the complete-case analysis preserves the type I error, the complete-case analysis and the covariate-only method have similar power, because both methods do not incorporate information of the auxiliary variables. As expected, the proposed method utilizes information about the missing data contained in the auxiliary variables and tends to yield higher power than the covariate-only method. The power gain from the incorporation of auxiliary variables can be small or even negative when the number of auxiliary variables $p$ is much larger than the (effective) sample size $\sum_{i=1}^{n} R_{i}$. In this case, the variable selection procedure cannot effectively identify the 
relevant auxiliary variables. This results in the inclusion of many noise variables into the working model of $S$, which in turn results in a worse fit than the covariate-only model that has no noise variables.

The true model method tends to have high power, because it uses the true model of S. Nevertheless, it is less powerful than the proposed method in some scenarios under Setting 2. This is because the true model contains many auxiliary variables with weak signals, and the extra information contained in the variables does not compensate the variability involved in the estimation of their effects. This illustrates that even when the true model is known, it may be desirable to perform variable selection and retain only the variables with strong signals.

\section{A real study}

We analyzed a dataset of patients with colorectal adenocarcinoma from TCGA (The Cancer Genome Atlas Network, 2012), available at http://gdac.broadinstitute.org/. In the study, demographic and clinical data, including age at diagnosis, sex, and tumor stage, as well as genomic data, including the expressions of RNA and protein, were measured. After removing subjects with missing clinical data, the sample size is 600 . The expressions of 18,068 genes, measured by RNA sequencing, are available for most subjects. The expressions of 204 proteins or phospho-proteins are available for only $78.2 \%$ of the subjects.

We focused on the association between individual protein expressions and tumor stage. We set the outcome variable to be tumor stage, dichotomized into stage I/II and stage III/IV, with respective proportions of 0.56 and 0.44 . In a single analysis, we set the 


\section{Setting 1}

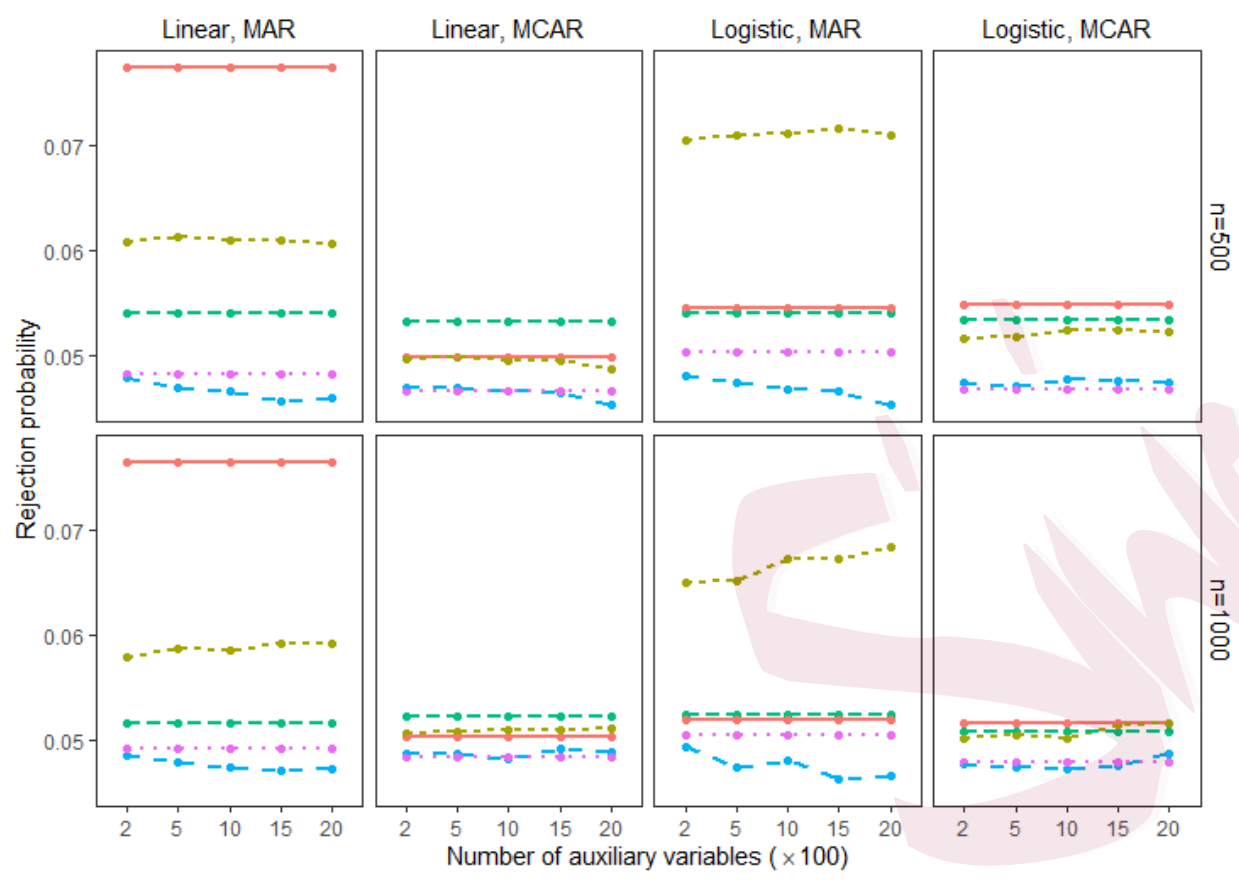

Setting 2
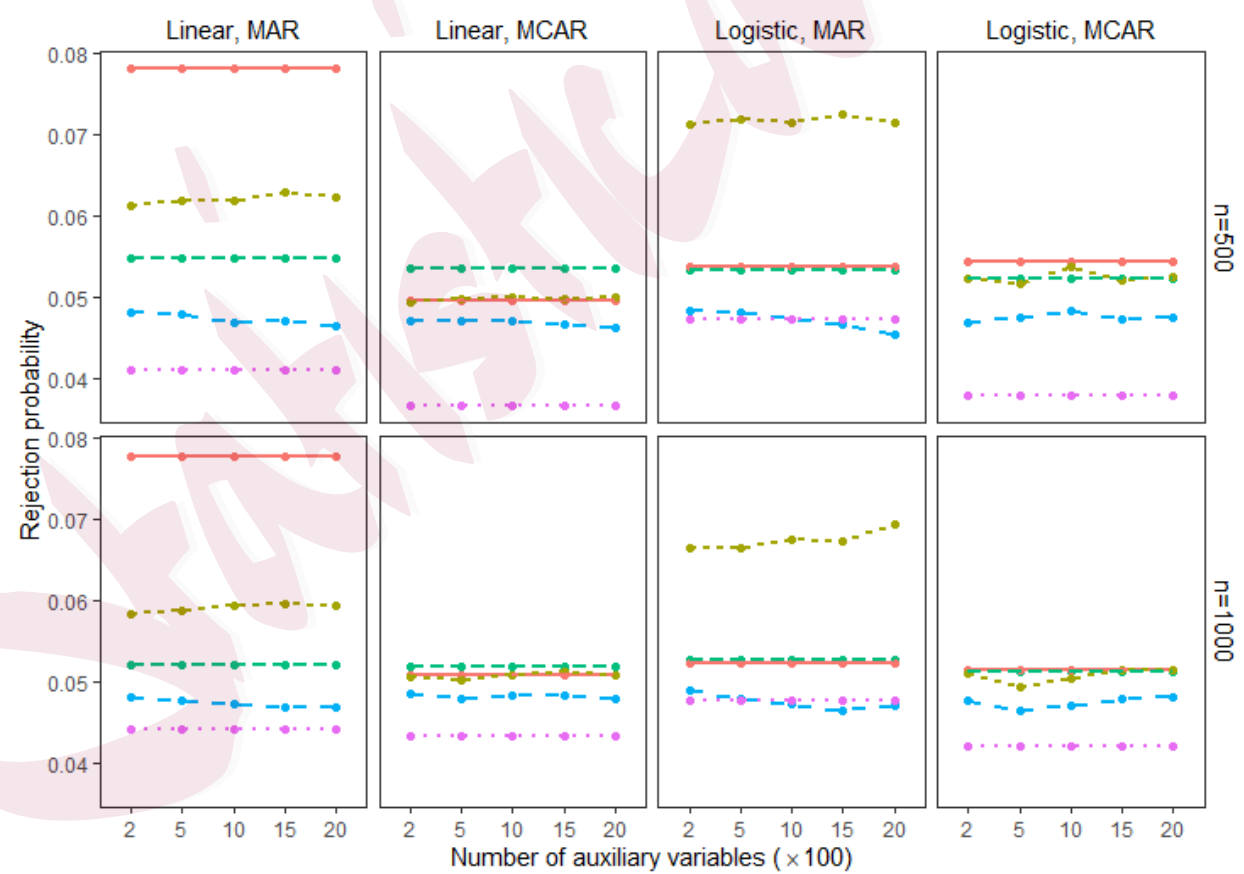

- Complete-case - - Simple imputation - - Covariate-only - - Proposed method … True model

Figure 2: Rejection probabilities under a missing proportion of $60 \%$ and the null hypothesis. 
Setting 1

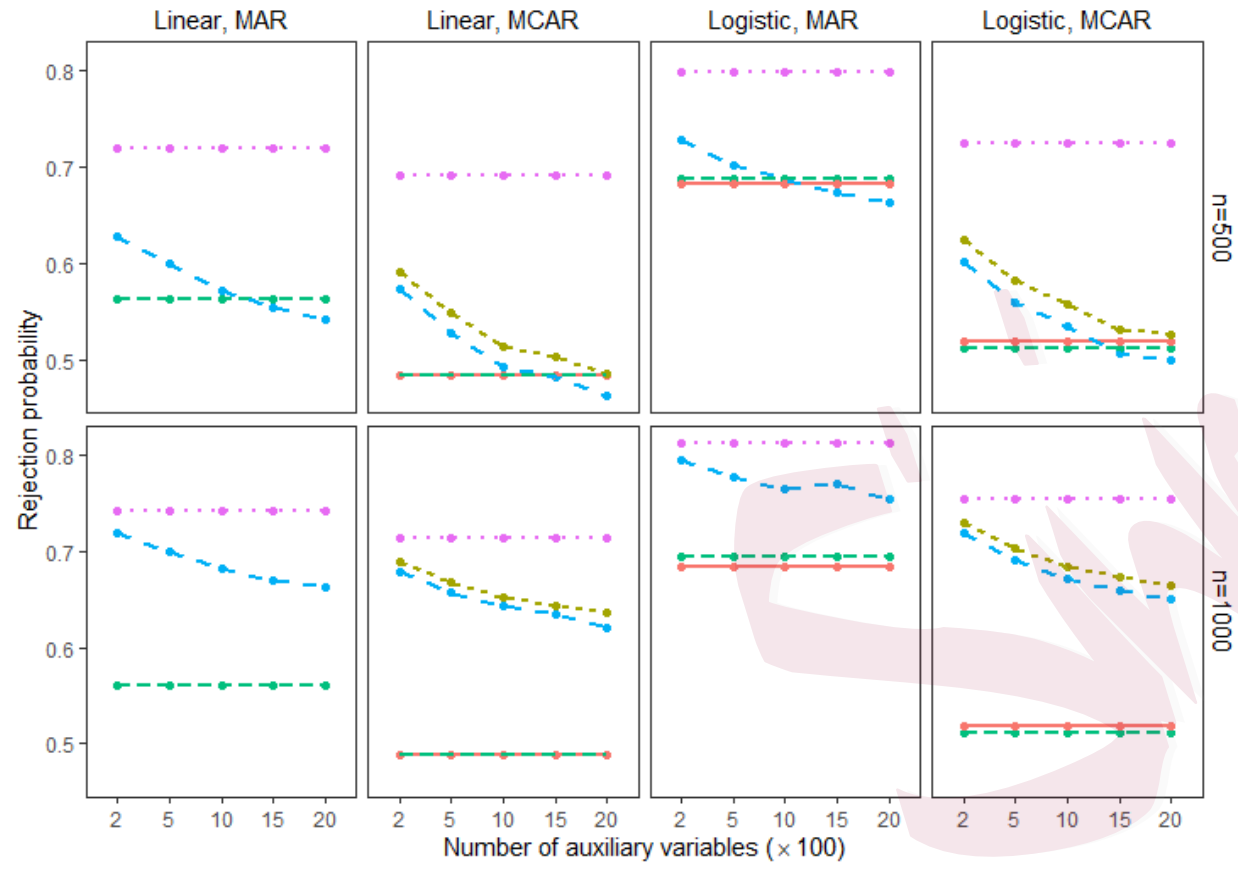

Setting 2

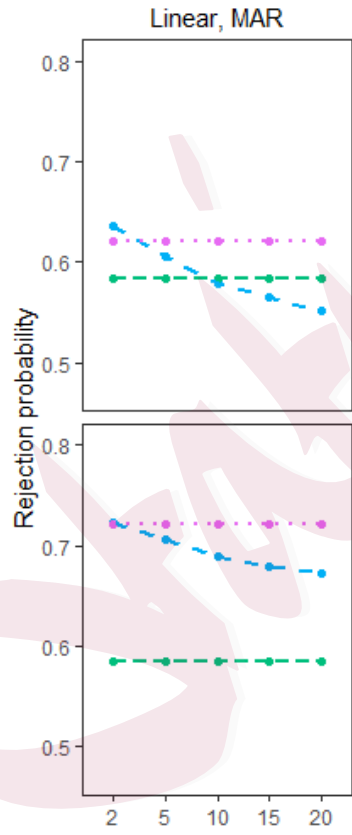

Linear, MCAR

Logistic, MAR
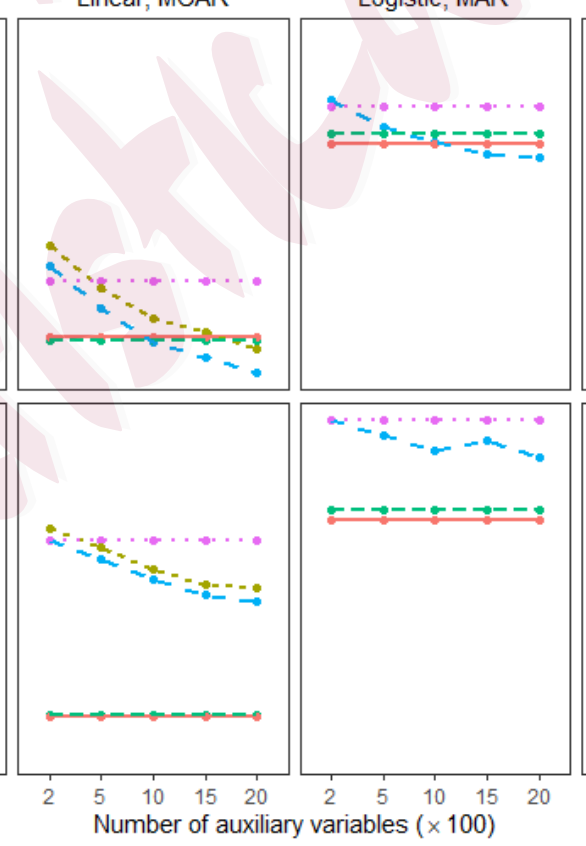

끙

공

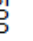

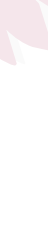
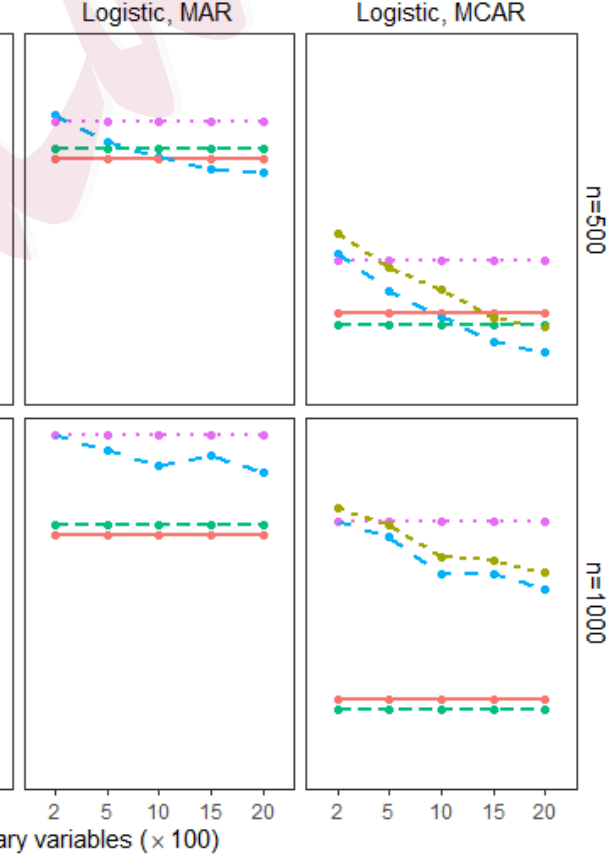

- Complete-case -- Simple imputation - - Covariate-only - - Proposed method $\cdots$ True model

Figure 3: Rejection probabilities under a missing proportion of $60 \%$ and the alternative hypothesis. 
covariate of interest $S$ to be the expression of a protein or phospho-protein. We set sex and age at diagnosis to be the covariates in $\boldsymbol{X}$ and set gene expressions as auxiliary variables. In the resulting model, $\beta$ represents the association between a protein and tumor stage for subjects with given age and sex. Note that the auxiliary variables are plausibly independent of $\boldsymbol{X}$, as required by condition (C4). The gene expression data are incomplete, and we impute the missing values using $k$-nearest neighbor imputation with $k=10$. We set the auxiliary variables $\boldsymbol{A}$ to be the top 200 principal components of the gene expressions; they appear to be more predictive than the individual gene expressions. We performed the proposed test with the working model of $S$ selected by the correlationbased marginal screening procedure in the simulation studies, and the screening threshold was selected by BIC. For comparison, we performed the score test using complete data only and the covariate-only method described in the simulation studies.

A total of 46 proteins were identified to be significantly associated with tumor stage at $\alpha=0.05$ under at least one of the three tests. Among the significant proteins, $76 \%$ have smaller $p$-values under the proposed method than the complete-case analysis, and $78 \%$ have smaller $p$-values under the proposed method than the covariate-only method. Many of the proteins that are more significant under the proposed method have been identified to be related to the progression of colorectal adenocarcinoma; the significant proteins and some relevant references are given in Table S1 of the Supplementary Material. This suggests that the proposed method is more powerful than the other two methods.

To investigate whether the power gain stems from the auxiliary variables, we inspect the relationship between the significance level and the variation explained by the gene expressions in the protein models. For a given protein, we let $Z_{1}$ and $Z_{2}$ be the indicators 


\section{DISCUSSION}

of whether the proposed method yields a smaller $p$-value than the complete-case analysis and the covariate-only method, respectively. Let $R^{2}$ be the coefficient of partial determination of the gene expressions, that is, the percentage of variation explained by the gene expressions given that sex and age are in the model. Among the significant proteins, the sample correlation between $Z_{1}$ and $R^{2}$ and that between $Z_{2}$ and $R^{2}$ are 0.32 and 0.22 , respectively. In addition, we classify each protein into one of two groups based on whether it is more significant under the proposed method than the complete-case analysis. Then, we test the difference in mean of $R^{2}$ between the two groups using the two-sample Wilcoxon test, and the $p$-value is 0.0381 . A similar analysis comparing the proposed method and the covariate-only method yields a $p$-value of 0.1271 . The results suggest that proteins with better fit of the imputation model tend to have higher power gain, especially when compared with the complete-case analysis.

\section{Discussion}

In this paper, we consider the association test between an outcome variable and an incomplete covariate, where the missing covariate values could be imputed using highdimensional auxiliary variables. We propose a simple two-step procedure that does not involve accounting for the variability of model selection in the first step and prove that such a procedure is asymptotically valid. This is in contrast with the conventional statistical intuition that standard inferential procedures on selected models are invalid and proper adjustments are needed (Fithian, Sun and Taylor, 2014; Lee et al., 2016). In the current setting, the model that involves variable selection is only of secondary interest. Although the fit of this model would affect the power of the test, the variability of model 


\section{DISCUSSION}

selection does not affect the asymptotic distribution of the score statistic.

A linear working model is assumed for the incomplete covariate $S$, but the validity of the score test does not depend on the correctness of this model. In fact, as demonstrated in the simulation studies, a simple working model may yield higher power than the true model when the latter is complex and involves many unknown parameters. Nevertheless, we require $S$ to exhibit a linear association with the low-dimensional covariates $\boldsymbol{X}$ in the outcome model. To relax this assumption, one may instead assume a nonparametric association between $S$ and $\boldsymbol{X}$ (Derkach, Lawless and Sun, 2015).

We focus on the asymptotic property of the score test under the null hypothesis. Evaluation of the asymptotic power of the test under contiguous alternatives is highly challenging, because the power depends on specifics of the model selection operator. The evaluation is even more complicated when $R$ depends on $Y$, in which case the missing mechanism for the data on $(S, \boldsymbol{X}, \boldsymbol{A})$ is not at random. To provide some insights to the power gain from the auxiliary variables, we evaluate the power under prespecified, fixeddimensional sets of auxiliary variables in the Supplementary Material. Under missing completely at random, inclusion of more auxiliary variables always increases the (asymptotic) power. Although the power generally does not have a simple form under missing at random, numerical evaluations suggest that the power tends to increase with the number of auxiliary variables. One should note, however, that these results are asymptotic and may not apply when the number of auxiliary variables is large compared to the sample size.

Our work can be extended in several directions. First, one may consider more general outcome models. In cancer genomic studies such as TCGA, some outcomes of interest 
6. DISCUSSION

are (possibly censored) time to events, such as time to cancer progression or death. It is of interest to consider semiparametric survival models for univariate or recurrent event times.

Second, in the current work, only a low-dimensional subset of auxiliary variables are used to impute the missing data, and the imputation model is fitted using least-squares estimation. It is of interest to consider a general imputation procedure that involves many auxiliary variables based on some regularized estimators, such as lasso, elastic net, and boosting. Such imputation procedures may be more accurate when many auxiliary variables are weakly associated with the incomplete covariate. The theoretical development would be highly challenging, because the regularized estimators may not have closed-formed expressions, and the dimension of the working model could be high.

Third, we have focused on hypothesis testing, and the theoretical results are developed under the null hypothesis. One may consider estimation and inference of the outcome model. In this case, the two-step procedure is invalid, because the missing mechanism would depend on $S$ through its dependence with $Y$, and estimation of the model of $S$ using only the subjects with observed data would be inconsistent. Also, one generally needs to account for the selection variability of the model of $S$ using the methods of, for example, Taylor and Tibshirani (2018).

\section{Supplementary Material}

The online Supplementary Material provides a discussion of model selection under marginal screening, evaluation of power, proofs of technical results, as well as a table and two figures presenting additional simulation and data analysis results. 


\section{DISCUSSION}

\section{Acknowledgements}

This research was supported by the Hong Kong Research Grants Council grant PolyU 253042/18P.

\section{Appendix 1: Relaxation of condition (C4)}

Let $\mathcal{M}_{n} \equiv\left\{j: j \in \mathcal{K}\right.$ for some $\left.\mathcal{K} \in \Omega_{n}\right\}$ be the collection of all "possibly selected" auxiliary variables and $\mathcal{M}_{n}^{\mathrm{C}}$ be its complement. Let $\mathcal{S}$ and $\mathcal{X}$ be the vector or matrix of the values of $S_{i}$ and $\boldsymbol{X}_{i}$ for subjects with $R_{i}=1$ as defined in Section 2 , and $\mathcal{A}_{\mathcal{M}_{n}}$ be the matrix that consists of rows of $\left\{\boldsymbol{A}_{\mathcal{M}_{n}, i}: R_{i}=1\right\}$. For any given $\left(\mathcal{S}, \mathcal{X}, \mathcal{A}_{\mathcal{M}_{n}}\right)$, let $\mathcal{K}\left(\mathcal{S}, \mathcal{X}, \mathcal{A}_{\mathcal{M}_{n}}\right)$ be the collection of models that could be selected under the given data values, that is,

$\mathcal{K}\left(\mathcal{S}, \mathcal{X}, \mathcal{A}_{\mathcal{M}_{n}}\right)=\left\{\mathcal{K}: \mathcal{K}^{*}\left\{\mathcal{S}-\mathcal{X} \widehat{\gamma}_{X},\left(\mathcal{A}_{\mathcal{M}_{n}}, \widetilde{\mathcal{A}}_{\mathcal{M}_{n}^{\mathrm{C}}}\right)\right\}=\mathcal{K}\right.$ for some $\left.\widetilde{\mathcal{A}}_{\mathcal{M}_{n}^{\mathrm{C}}} \in \mathbb{R}^{\left(\sum_{i} R_{i}\right) \times\left(p_{n}-\left|\mathcal{M}_{n}\right|\right)}\right\}$.

For any $\mathcal{K} \in \Omega_{n}$, define

$$
\overline{\mathcal{K}}=\left\{\mathcal{M}: \mathcal{M} \in \mathcal{K}\left(\widetilde{\mathcal{S}}, \widetilde{\mathcal{X}}, \widetilde{\mathcal{A}}_{\mathcal{M}_{n}}\right) \text { for some }\left(\widetilde{\mathcal{S}}, \widetilde{\mathcal{X}}, \widetilde{\mathcal{A}}_{\mathcal{M}_{n}}\right) \text { such that } \mathcal{K} \in \mathcal{K}^{*}\left(\widetilde{\mathcal{S}}, \widetilde{\mathcal{X}}, \widetilde{\mathcal{A}}_{\mathcal{M}_{n}}\right)\right\}
$$

We can understand $\overline{\mathcal{K}}$ as the collection of models that are "close" to $\mathcal{K}$ : there exist auxiliary variable values $\widetilde{\mathcal{A}}_{\mathcal{M}_{n}}$ that are compatible with the selection of $\mathcal{K}$ as well as the selection of other elements of $\overline{\mathcal{K}}$. For marginal screening, because the selection of components of $\boldsymbol{A}_{\mathcal{M}_{n}}$ depends only on $\left(\mathcal{S}, \mathcal{X}, \mathcal{A}_{\mathcal{M}_{n}}\right)$ but not $\mathcal{A}_{\mathcal{M}_{n}^{C}}, \overline{\mathcal{K}}$ consists of models that include variables in $\mathcal{K}$ along with a subset of variables in $\boldsymbol{A}_{\mathcal{M}_{n}^{\mathrm{C}}}$.

We can replace condition (C4) in Theorems 1 and 2 and Corollary 1 by 


\section{DISCUSSION}

(C4') Under $\beta=0$, the residual $\left(S-\gamma_{0 X}^{\mathrm{T}} \boldsymbol{X}\right)$ and the covariate $\boldsymbol{X}$ are independent, and $\boldsymbol{A}_{\mathcal{M}_{n}}$ is independent of $(Y, \boldsymbol{X})$. Also, $\sum_{\mathcal{K} \in \Omega_{n}} P\left(\mathcal{K}^{*} \neq \mathcal{K}, \mathcal{K}^{*} \in \overline{\mathcal{K}}\right) \rightarrow 0$.

For marginal screening, elements of $\left\{\overline{\mathcal{K}}: \mathcal{K} \in \Omega_{n}\right\}$ are mutually exclusive. The second part of condition ( $\mathrm{C}^{\prime}$ ') is automatically satisfied, because

$$
\sum_{\mathcal{K} \in \Omega_{n}} P\left(\mathcal{K}^{*} \neq \mathcal{K}, \mathcal{K}^{*} \in \overline{\mathcal{K}}\right)=P\left(\bigcup_{\mathcal{K} \in \Omega_{n}}\left\{\mathcal{K}^{*} \neq \mathcal{K}, \mathcal{K}^{*} \in \overline{\mathcal{K}}\right\}\right) \leq P\left(\mathcal{K}^{*} \notin \Omega_{n}\right) \rightarrow 0
$$

\section{Appendix 2: Outline of the proof of Theorem 1}

We outline the proof of Theorem 1 in this Appendix and relegate the complete proof to the Supplementary Material. By a version of the portmanteau theorem (Pollard, 2002, p. 177), it suffices to prove that for any function $g$ with bounded derivatives up to the third order,

$$
\mathrm{E}\left[g\left\{\frac{U_{\beta}\left(\widehat{\boldsymbol{\alpha}}, \widehat{\boldsymbol{\gamma}}_{\mathcal{K}^{*}}\right)}{\sigma\left(\mathcal{K}^{*}\right)}\right\}\right] \rightarrow \mathrm{E}\{g(Z)\}
$$

where $Z$ is a standard normal variable. The first step of the proof is to expand $U_{\beta}\left(\widehat{\boldsymbol{\alpha}}, \widehat{\gamma}_{\mathcal{K}}\right)$

$$
\begin{aligned}
& \frac{1}{n^{1 / 2}} \sum_{i=1}^{n}\left\{\epsilon_{i}-\mathrm{E}\left(\epsilon \mid R_{i}, \boldsymbol{X}_{i}\right)\right\}\left\{R_{i} S_{i}+\left(1-R_{i}\right) \boldsymbol{\gamma}_{0 \mathcal{K}}^{\mathrm{T}} \boldsymbol{W}_{\mathcal{K}, i}+\boldsymbol{I}_{\beta \alpha}^{\mathrm{T}} \boldsymbol{I}_{\alpha \alpha}^{-1} \boldsymbol{X}_{i}\right\} \\
& +\frac{1}{n^{1 / 2}} \sum_{i=1}^{n}\left[\left(\gamma_{0 X}^{\mathrm{T}}+\boldsymbol{I}_{\beta \alpha}^{\mathrm{T}} \boldsymbol{I}_{\alpha \alpha}^{-1}\right)\left\{\mathrm{E}\left(\epsilon \mid R_{i}, \boldsymbol{X}_{i}\right) \boldsymbol{X}_{i}-\mathrm{E}\left(\epsilon \boldsymbol{X} \mid R_{i}\right)\right\}\right. \\
& \left.+\left\{\mathrm{E}\left(\epsilon \mid R_{i}, \boldsymbol{X}_{i}\right)-\mathrm{E}\left(\epsilon \mid R_{i}\right)\right\} \boldsymbol{\gamma}_{0 A, \mathcal{K}}^{\mathrm{T}} \boldsymbol{A}_{\mathcal{K}, i}+\left\{\mathrm{E}\left(\epsilon \mid R_{i}, \boldsymbol{X}_{i}\right)+\boldsymbol{I}_{\beta \gamma}^{\mathrm{T}} \boldsymbol{I}_{\gamma \gamma}^{-1} \boldsymbol{W}_{\mathcal{K}, i}\right\} R_{i}\left(S_{i}-\boldsymbol{\gamma}_{0 \mathcal{K}}^{\mathrm{T}} \boldsymbol{W}_{\mathcal{K}, i}\right)\right] \\
& +\frac{1}{n^{1 / 2}} \sum_{i=1}^{n}\left\{\left(\gamma_{0 X}^{\mathrm{T}}+\boldsymbol{I}_{\beta \alpha}^{\mathrm{T}} \boldsymbol{I}_{\alpha \alpha}^{-1}\right) \mathrm{E}\left(\epsilon \boldsymbol{X} \mid R_{i}\right)+\mathrm{E}\left(\epsilon \mid R_{i}\right) \boldsymbol{\gamma}_{0 A, \mathcal{K}}^{\mathrm{T}} \boldsymbol{A}_{\mathcal{K}, i}\right\}+o_{p}(1) \\
& \equiv \frac{1}{n^{1 / 2}} \sum_{i=1}^{n} U_{1 i}(\mathcal{K})+\frac{1}{n^{1 / 2}} \sum_{i=1}^{n} U_{2 i}(\mathcal{K})+\frac{1}{n^{1 / 2}} \sum_{i=1}^{n} U_{3 i}(\mathcal{K})+o_{p}(1),
\end{aligned}
$$




\section{DISCUSSION}

where the $o_{p}(1)$ terms converge in mean to zero uniformly over $\mathcal{K} \in \Omega_{n}$. As a result, the left-hand side of $(6.3)$ can be written as

$$
\int_{\mathcal{K} \in \Omega_{n}} \mathrm{E}\left[g\left\{n^{-1 / 2} \sum_{i=1}^{n} \frac{U_{1 i}(\mathcal{K})+U_{2 i}(\mathcal{K})+U_{3 i}(\mathcal{K})}{\sigma(\mathcal{K})}\right\} \mid \mathcal{K}^{*}=\mathcal{K}\right] \mathrm{d} \mathcal{P}_{\mathcal{K}^{*}}(\mathcal{K})+o(1)
$$

where $\mathcal{P}_{\mathcal{K}^{*}}$ is the probability measure of $\mathcal{K}^{*}$.

The main argument of the proof is to show that $n^{-1 / 2} \sum_{i=1}^{n} U_{k i}(\mathcal{K})$ for $k=1,2,3$ in (6.4) can in turn be replaced by normal variables. Note that conditional on $\mathcal{O}_{1} \equiv$ $\left(R_{i}, S_{i}, \boldsymbol{W}_{\mathcal{K}, i}\right)_{i=1, \ldots, n}, U_{11}(\mathcal{K}), \ldots, U_{1 n}(\mathcal{K})$ are mean zero and independent. For $i=1, \ldots, n$ let

$$
\widetilde{U}_{1 i}(\mathcal{K})=\operatorname{Var}\left(\epsilon \mid R_{i}, \boldsymbol{X}_{i}\right)^{1 / 2}\left\{R_{i} S_{i}+\left(1-R_{i}\right) \boldsymbol{\gamma}_{0 \mathcal{K}}^{\mathrm{T}} \boldsymbol{W}_{\mathcal{K}, i}+\boldsymbol{I}_{\beta \alpha}^{\mathrm{T}} \boldsymbol{I}_{\alpha \alpha}^{-1} \boldsymbol{X}_{i}\right\} Z_{1 i}
$$

where $Z_{11}, \ldots, Z_{1 n}$ are i.i.d. standard normal random variables that are independent of the observed data. Because the first and second moments of $U_{1 i}$ and $\widetilde{U}_{1 i}$ given $\mathcal{O}_{1}$ match and $\left\{\mathcal{K}^{*}=\mathcal{K}\right\}$ is implied by $\mathcal{O}_{1}$, the moments given $\left\{\mathcal{K}^{*}=\mathcal{K}\right\}$ also match. We then use Lindeberg's telescoping argument for the central limit theorem (Chung, 2001, p. 211) to show that $n^{-1 / 2} \sum_{i=1}^{n} U_{1 i}(\mathcal{K})$ in $(6.4)$ can be replaced by $n^{-1 / 2} \sum_{i=1}^{n} \widetilde{U}_{1 i}(\mathcal{K})$. We further show that the term can be replaced by a normal variable with mean zero and variance $\sigma_{1}^{2}(\mathcal{K})$

Next, we show that under condition (C5), the event $\left\{\mathcal{K}^{*}=\mathcal{K}\right\}$ in the conditional expectation in 6.4 can be replaced by $\left\{\mathcal{K}_{0}^{*}=\mathcal{K}\right\}$, where $\mathcal{K}_{0}^{*} \equiv \mathcal{K}_{0}^{*}\left(\mathcal{S}-\mathcal{X} \gamma_{0 X}, \mathcal{A}\right)$ is the selected model based on the actual residual $\left(S-\gamma_{0 X}^{\mathrm{T}} \boldsymbol{X}\right)$. Then, we note that $\left\{\mathcal{K}_{0}^{*}=\mathcal{K}\right\}$ is implied by $\mathcal{O}_{2} \equiv\left(R_{i}, \boldsymbol{A}_{i}, S_{i}-\boldsymbol{\gamma}_{0 X}^{\mathrm{T}} \boldsymbol{X}_{i}\right)_{i=1, \ldots, n}$, and conditional on $\mathcal{O}_{2}, U_{21}(\mathcal{K}), \ldots, U_{2 n}(\mathcal{K})$ are mean zero and independent; under this conditional probability space, the random 
element in $U_{2 i}(\mathcal{K})$ is $\boldsymbol{X}_{i}$. We can similarly show that $n^{-1 / 2} \sum_{i=1}^{n} U_{2 i}(\mathcal{K})$ in 6.4 can be replaced by a normal variable with mean zero and variance $\sigma_{2}^{2}(\mathcal{K})$.

Finally, we show that after $n^{-1 / 2} \sum_{i=1}^{n} U_{1 i}(\mathcal{K})$ and $n^{-1 / 2} \sum_{i=1}^{n} U_{2 i}(\mathcal{K})$ are replaced by normal variables, the conditional expectation in 6.4 can be replaced by a marginal expectation under condition $(\mathrm{C} 6)$. It is easy to see that $U_{31}(\mathcal{K}), \ldots, U_{3 n}(\mathcal{K})$ are mean zero and independent, and thus $n^{-1 / 2} \sum_{i=1}^{n} U_{3 i}(\mathcal{K})$ can be replaced by a normal variable with mean zero and variance $\sigma_{3}^{2}(\mathcal{K})$. Combining the above results, we conclude that the variable in the function $g$ in 6.4 can be replaced by a standard normal variable, and the desired result follows.

In the conventional argument for the asymptotic distribution of the score statistic, we expand $U_{\beta}\left(\widehat{\boldsymbol{\alpha}}, \widehat{\gamma}_{\mathcal{K}}\right)$ as in $(2.2)$, and the asymptotic normality of the score statistic (given $\boldsymbol{X}$ and $\boldsymbol{A}$ ) follows from the central limit theorem. However, conditional on the model selection event $\left\{\mathcal{K}^{*}=\mathcal{K}\right\},\left(S_{i}-\gamma_{0 \mathcal{K}}^{\mathrm{T}} \boldsymbol{W}_{\mathcal{K}, i}\right)_{i=1, \ldots, n}$ are dependent, and the central limit theorem does not apply. In our proof, instead of relying on the independence of $\left(S_{i}-\boldsymbol{\gamma}_{0 \mathcal{K}}^{\mathrm{T}} \boldsymbol{W}_{\mathcal{K}, i}\right)$ 's, we establish the asymptotic normality based on the (conditional) independence and mean-zero property of functions of $\boldsymbol{X}_{i}$ 's given the model selection event.

\section{References}

Bachoc, F., Leeb, H. and Pötscher, B. M. (2019). Valid confidence intervals for post-model-selection predictors. The Annals of Statistics 47, 1475-1504.

Berk, R., Brown, L., Buja, A., Zhang, K. and Zhao, L. (2013). Valid post-selection inference. The Annals of Statistics 41, 802-837.

Bjørnland, T., Bye, A., Ryeng, E., Wisløff, U. and Langaas, M. (2018). Powerful extreme phenotype sampling 
designs and score tests for genetic association studies. Statistics in Medicine 37, 4234-4251.

Chung, K. L. (2001). A Course in Probability Theory (3rd ed.). Academic Press.

Derkach, A., Lawless, J. F. and Sun, L. (2015). Score tests for association under response-dependent sampling designs for expensive covariates. Biometrika 102, 988-994.

Fan, J. and Lv, J. (2008). Sure independence screening for ultrahigh dimensional feature space. Journal of the Royal Statistical Society: Series B 70, 849-911.

Fan, J. and Song, R. (2010). Sure independence screening in generalized linear models with NP-dimensionality. The Annals of Statistics 38, 3567-3604.

Fithian, W., Sun, D. and Taylor, J. (2014). Optimal inference after model selection. Preprint. Available at arXiv:1410.2597.

Heller, R., Chatterjee, N., Krieger, A. and Shi, J. (2018). Post-selection inference following aggregate level hypothesis testing in large-scale genomic data. Journal of the American Statistical Association 113, 17701783.

Hu, Y.-J., Li, Y., Auer, P. L. and Lin, D. Y. (2015). Integrative analysis of sequencing and array genotype data for discovering disease associations with rare mutations. Proceedings of the National Academy of Sciences $\mathbf{1 1 2}$, 1019-1024.

Kuchibhotla, A. K., Brown, L. D., Buja, A., Cai, J., George, E. I. and Zhao, L. H. (2020). Valid post-selection inference in model-free linear regression. The Annals of Statistics 48, 2953-2981.

Lawless, J. (2018). Two-phase outcome-dependent studies for failure times and testing for effects of expensive covariates. Lifetime Data Analysis 24, 28-44.

Lee, J. D., Sun, D. L., Sun, Y. and Taylor, J. E. (2016). Exact post-selection inference, with application to the lasso. The Annals of Statistics 44, 907-927. 


\section{REFERENCES}

Little, R. J. and Rubin, D. B. (2019). Statistical Analysis With Missing Data (3rd ed.). John Wiley \& Sons.

Ning, Y. and Liu, H. (2017). A general theory of hypothesis tests and confidence regions for sparse high dimensional models. The Annals of Statistics 45, 158-195.

Pollard, D. (2002). A User's Guide to Measure Theoretic Probability. Number 8. Cambridge University Press.

Prentice, R. L. and Pyke, R. (1979). Logistic disease incidence models and case-control studies. Biometrika 66, 403-411.

Taylor, J. and Tibshirani, R. (2018). Post-selection inference for $\ell_{1}$-penalized likelihood models. Canadian Journal of Statistics 46, 41-61.

The Cancer Genome Atlas Network (2012). Comprehensive molecular characterization of human colon and rectal cancer. Nature 487, 330-337.

Tian, X., Loftus, J. R. and Taylor, J. E. (2018). Selective inference with unknown variance via the square-root lasso. Biometrika 105, 755-768.

Tibshirani, R. (1996). Regression shrinkage and selection via the lasso. Journal of the Royal Statistical Society: Series B 58, 267-288.

Tibshirani, R. J., Taylor, J., Lockhart, R. and Tibshirani, R. (2016). Exact post-selection inference for sequential regression procedures. Journal of the American Statistical Association 111, 600-620.

van de Geer, S., Bühlmann, P., Ritov, Y. and Dezeure, R. (2014). On asymptotically optimal confidence regions and tests for high-dimensional models. The Annals of Statistics 42, 1166-1202.

Wong, K. Y., Zeng, D. and Lin, D. Y. (2019). Robust score tests with missing data in genomics studies. Journal of the American Statistical Association 114, 1778-1786.

Zhang, C.-H. and Zhang, S. S. (2014). Confidence intervals for low dimensional parameters in high dimensional linear models. Journal of the Royal Statistical Society: Series B 76, 217-242. 


\section{Kin Yau Wong}

Department of Applied Mathematics, The Hong Kong Polytechnic University, Hong Kong

E-mail: kin-yau.wong@polyu.edu.hk

Jiahui Feng

Department of Applied Mathematics, The Hong Kong Polytechnic University, Hong Kong

E-mail: jia-hui.feng@connect.polyu.hk 